\title{
Oscillating Agent Model in Terms of Adapted Orch OR Theory
}

\author{
Darius Plikynas* \\ Research and Development Center \\ KSU \\ Vilnius, Lithuania, EU \\ *Corresponding author
}

\begin{abstract}
In this multidisciplinary paper, there are some explanatory sources provided that pertain to the oscillating agent model (OAM), which is an intrinsic part of the novel oscillationsbased multiagent system (OSIMAS) paradigm. The agent's oscillating nature is inferred by empirical neuroscience observations. In the OAM, a social agent is understood as a set of characteristic basic mind-brain states (BMS) and transitions between them. The neural correlations of consciousness are fundamentally backed-up using an adaption of the HameroffPenrose Orch OR quantum theory of neuronal beats, which has recently been confirmed by some experimental studies on neural cells' activities in microtubules. This paper provides a putative link between the Orch OR, a conceptual oscillations-based OSIMAS framework, and the empirically observed coherent bioelectromagnetic oscillations of ensembles of neurons in the brain (EEG spectra). It also sheds new light on the novel interpretation of the Orch OR theory of quantum beats. In this short work-in-progress report, a proposed multidisciplinary approach provides conceptual tools for the modeling of the new type of agency.
\end{abstract}

Keywords-oscillating agent model; quantum beats; EEG spectra; brainwaves; basic mind states

\section{INTRODUCTION}

Enabled by recent neuroscience studies, this paper further extends the conceptually novel modeling approach of basic human mind states (BMS) as systems of coherent oscillations. That is why this approach is called the oscillating agent model (OAM), which is an intrinsic part of the novel oscillationsbased multiagent system (OSIMAS) paradigm. The final goal of the OSIMAS is to build a multidisciplinary simulation framework for the new kind of agency and multi-agent system, e.g., [1]. In this scheme, the OAM is aimed at modeling a single agent, bridging the gap between fundamental theories, neuroscience-based observations and simulations.

The quantum beats approach described in this paper further underpins our earlier research, where we explored various other possibilities of employing phonons, the coupled oscillators energy exchange model (adapted Kuramoto model), wave functions and quantum operators for a stylized description of basic mind states (BMS) and transitions between them [2]-[4].

In the OAM, BMS are defined using experimentally observed EEG spectra, i.e., brainwaves (delta, theta, alpha, beta and gamma spectral ranges), which in an empirical manner, reveal the oscillatory nature of the agent's mind states. Such an approach provides an opportunity to fine tune the model according to the experimental data. In this way, the dynamics of transitions between BMS can be stylized using oscillationsbased representations of the characteristic EEG power spectral density (PSD) distributions of brainwaves observed in the real measurements of mind-brain states. Our recent studies pertain to this line of research [5], [6].

From a wider perspective, the mind-brain-body issue has a long history of multidisciplinary investigation. Recently, many research frameworks have been proposed to reconcile the mental and the quantum (physical) states. For an introductory purpose, a few of the general concepts on brain functioning based on recent neurobiological investigations and quantum mechanical approaches are briefly mentioned below (albeit, quantum theoretical formalism is realized in a nonliving context [7]).

A recent review of the quantum modeling of mental states was made by Meijer and Korf [8]. They noted that some quantum mechanical (QM) theories have been justified by their basic elements of (i) uncertainty, (ii) a universal consciousness with the individual consciousness as a participating agent, (iii) consciousness, even seen as a non-physical entity, acting in the physical domain with a causal power, (iv) explaining the mental transitions between states, etc.

As we can notice, QM theories can potentially open a vast new field of research providing a totally novel standpoint towards mental phenomena and agency as well; quantum theories that see the mind defined by energy/fields to describe and understand the mental aspects of reality [7], [9]-[11].

It is also important to note that the neurobiological and QM concepts may function in a complementary fashion. The classical neurological interpretation of the brain can therefore be seen as a flow of information between molecular and neuronal networks, whereas quantum approaches are based on more fundamental atomic and subatomic coherent and entangled states, implying a non-local and unbroken reality. In that regard, it closely resembles the OSIMAS approach, which uses a pervasive information field (PIF) concept (Plikynas et al., 2014).

\section{ORCH OR: THE QUANTUM BEATS APPROACH}

In the light of the other QM concepts, theories and applications, a conceptually novel mind states modeling 
approach is presented. It aims to bridge recent neuroscience discoveries with the classical physics and QM theory. Hence, an oscillating agent model (OAM) construction using (i) OSIMAS premises [12] and (ii) the 'Orch OR' (orchestrated objective reduction) theory of consciousness proposed by Stuart Hameroff and Sir Roger Penrose (Hameroff \& Penrose, 2014b) is extended below.

Following the Orch OR theory, conscious states have been assumed to correlate with physiological EEG oscillations, which might come about, namely as beat frequencies, arising when OR (as it pertains to the specific Diósi-Penrose scheme of 'objective reduction' ('OR') of the quantum state [13]) is applied to the superposition of quantum states of slightly different energies.

In short, if there is a state $\Psi$ which is a superposition of two slightly different states $\Psi_{1}$ and $\Psi_{2}$, each of which would be stationary on its own, but with very slightly different respective energies $E_{1}$ and $E_{2}$, then the superposition would not be quite stationary. Its basic frequency would be the average $\left(E_{1}+E_{2}\right) / 2 \hbar$ of the two, corresponding to the average energy $1 / 2\left(E_{1}+E_{2}\right)$, but this would be modulated by a much lower classical frequency ('beats') that is the difference between the two, namely $\left|E_{1}-E_{2}\right| / h$, as follows, very roughly, from the following mathematical identity

$$
\varepsilon^{\mathrm{la}}+\varepsilon^{\mathrm{lb}}=2 \varepsilon^{\mathrm{l}(\mathrm{a}+\mathrm{b}) / 2} \chi O \sigma(\mathrm{a}-\mathrm{b}) / 2,
$$

where we may take $a=-E_{1} t / \hbar$ and $b=-E_{2} t / \hbar$ to represent the quantum wave functions for the two energies. The superposition of complex quantum oscillations has a frequency which is the average of the two, but this is modulated by a classical oscillation, as given by the cosine term with a much lower frequency determined by the difference between the quantum mechanical frequencies $E_{1}$ and $E_{2}$ of the two individual states $\Psi_{1}$ and $\Psi_{2}[13]$.

Thus, according to Orch OR, we may consider conscious moments occurring with beat frequencies $\left|E_{1}-E_{2}\right| / h$, rather than the primary frequencies of $E_{1} / h$ and $E_{2} / h$. The primary frequencies may be around 10 megahertz, with time periods of $\sim 10^{-8} \mathrm{~s}$. Decoherence might need be avoided for a mere tenmillionth of a second with consciousness occurring at far slower beat frequencies [13]. For example, if $E_{1} / h$ and $E_{2} / h$ were 10.000000 megahertz and 10.000040 megahertz respectively, a beat frequency of $40 \mathrm{~Hz}$ (by $\left|E_{l}-E_{2}\right| / h$ ) could correlate with discrete conscious moments.

Admittedly, a relatively high rate of discrete conscious moments at about $40 \mathrm{~Hz}$ is located in the EEG gamma brainwave band, which is mostly associated with the brain binding into a coherent system for the purpose of complex cognitive or motor functions. That is, intensive states of conscious alertness need higher rates of conscious moments, i.e., higher beat frequencies. However, in the restful or sleeping states, our brains do not need high conscious alertness. Therefore, in these states, the conscious moments produced by the beat frequencies become less frequent and stay in the alpha and theta spectral ranges. In the case of deep sleep, the conscious moments of alert awareness occur in the very infrequent delta spectral range [6].

\section{OAM: THE ADAPTED QUANTUM BEATS APPROACH}

The presented model extends the Orch OR theory, providing a novel interpretation for the quantum states $\Psi_{1}$ and $\Psi_{2}$. That is, the quantum state $\Psi_{1}$ is denoted as stationary primary state $\Psi_{p}$ with a respective energy of $E_{p}$ and a corresponding primary frequency of $\omega_{p}=E_{p} / h$. The other slightly different state is denoted as reflected $\Psi_{r}$ with a respective energy of $E_{r}$ and a corresponding primary frequency of $\omega_{r}=E_{r} / h$, see Figure 1 .

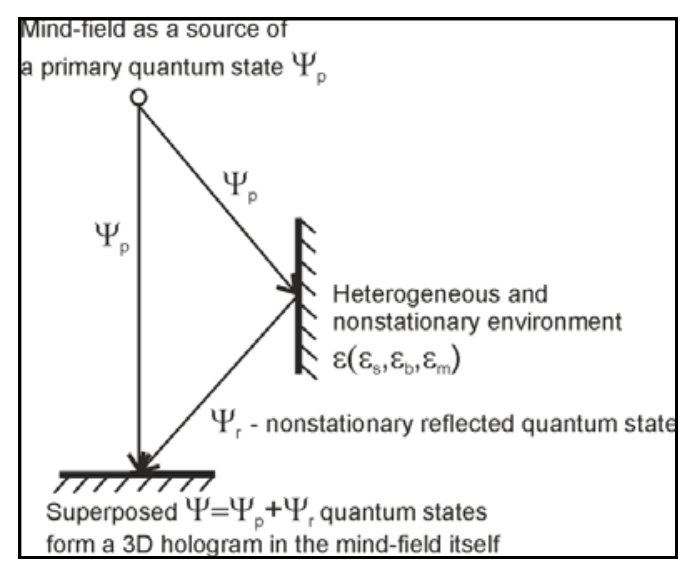

FIGURE I. SUPERPOSITION OF STATIONARY PRIMARY QUANTUM STATE $\Psi_{P}$ AND NONSTATIONARY REFLECTED STATE $\Psi_{R}$. HOLOGRAPHIC PRINCIPLE: 3D HOLOGRAM FORMATION IN THE MIND-FIELD

In principle, the superposition of stationary primary state $\Psi_{p}$ and nonstationary reflected state $\Psi_{r}$ proposed here can also be interpreted in holographic terms, see Figure 1. However, in this case, the interference of the reference and reflected (from the object) beams is not projected as usual in the $2 \mathrm{D}$ plate, but instead takes place in the 3D space of the mind-field. Besides, instead of the beams' interference, we are dealing with the superposition of the quantum functions or so-called quantum states.

Reflected state $\Psi_{r}$ is obtained as primary state's $\Psi_{p}$ reflection from environmental factors. In essence, the biological mechanism for the evolvement of primary state $\Psi_{p}$ and its energy and frequency correlates of consciousness has been explicitly described in the Stuart Hameroff and Sir Roger Penrose Orch OR theory (Hameroff \& Penrose, 2014) as quantum state $\Psi_{1}$. However, the Orch OR theory has little to say about nonstationary quantum state $\Psi_{2}$. It assumes the existence of some endogenous factors at the microtubules level that produce two slightly different quantum states, but it does not elaborate about why such differences occurs.

According to the OAM, nonstationary environment $\varepsilon$ is a major driving factor, which influences the emergence of nonstationary state $\Psi_{r}$ and, consequently, quantum superposed state $\Psi$. We maintain that environment $\varepsilon$ is heterogeneous, i.e., it embraces 1) the external sensory environment $\varepsilon_{s}, 2$ ) the body as an internal environment $\varepsilon_{b}$, and 3 ) the mind as the deepest internal environment $\varepsilon_{m}$ with stored emotions, feelings, memories, thinking patterns, etc. All these environments $\varepsilon^{=} \varepsilon$ $\left(\varepsilon_{s}, \varepsilon_{b}, \varepsilon_{m}\right)$ simultaneously affect the primary quantum state $\Psi_{p}$, 
producing reflected state $\Psi_{r}$ with complex EEG spectral energy patterns in the delta, theta, alpha, beta and gamma brainwave

ranges, see Figure 2.

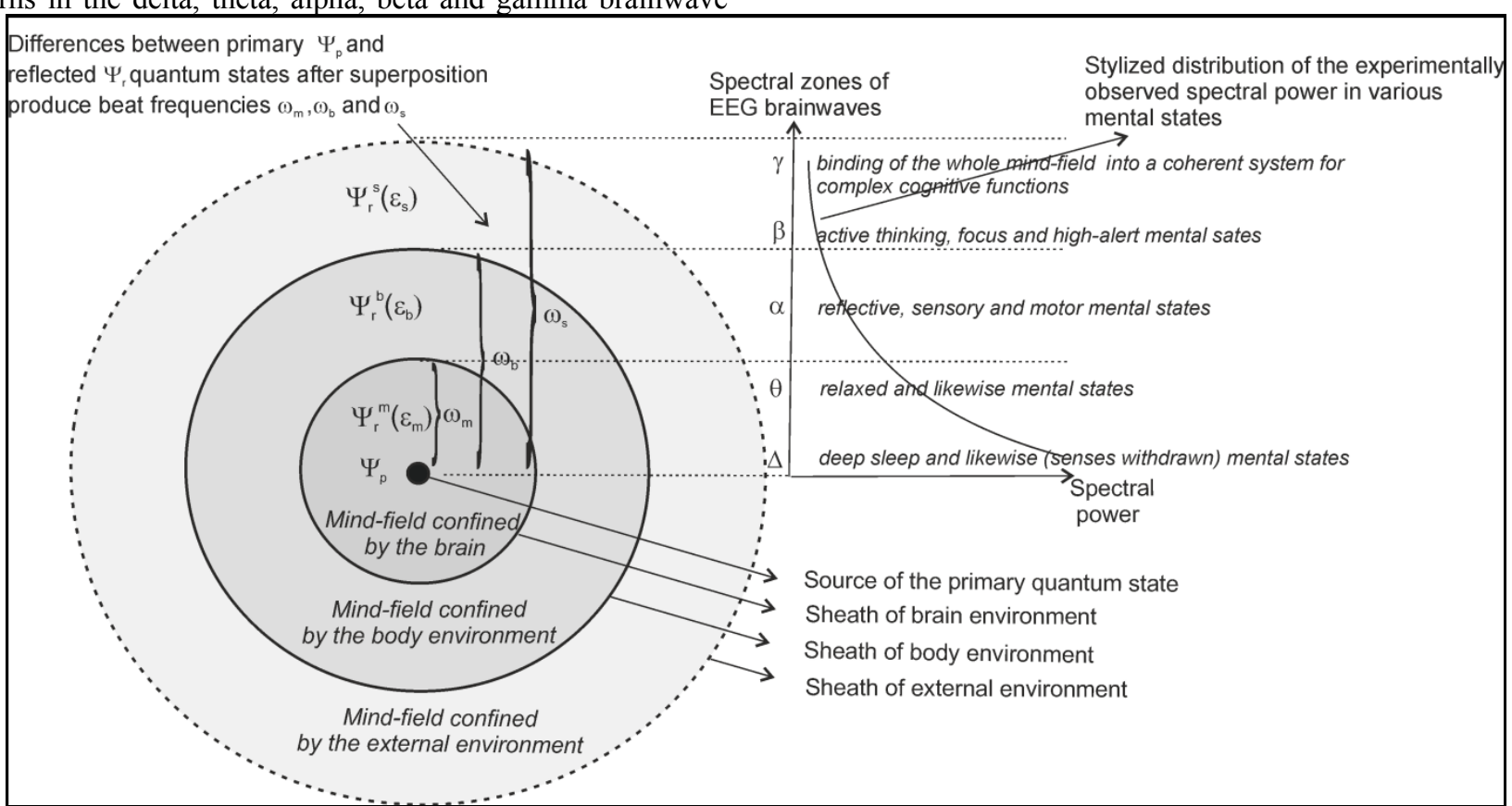

FIGURE II. FOLLOWING THE OAM AND ORCH OR CONCEPTUAL IDEAS, AN AGENT IS REPRESENTED AS A COGNITIVE PROCESS OF A SPATIALLY AND SPECTRALLY DISTRIBUTED MIND-FIELD THAT EMERGES AS A CONSEQUENCE OF THE SUPERPOSITION OF PRIMARY AND REFLECTED QUANTUM STATES. SUPPOSEDLY IT DIRECTLY CAUSES THE LOW FREQUENCY BEATS EMPIRICALLY REGISTERED AS BRAINWAVES

Following this line of thought, reflected state $\Psi_{r}$ can be interpreted as a high frequency (in the megahertz range) correlate of conscious awareness of the environment at the cellular level. Consequently, primary stationary state $\Psi_{p}$ interacts with its own reflected and slightly affected (by the environment) state $\Psi_{r}$. In this manner, superposed state $\Psi$ emerges. Like in the case of Orch OR, superposed state $\Psi$ has a frequency that is the average of the two $\left(\omega_{p}\right.$ and $\left.\omega_{r}\right)$, but this is modulated by a cos term with a much lower beat frequency determined by the difference between $\omega_{p}$ and $\omega_{r}$, see Eq. 1 .

Hence, superposed state $\Psi$ has two levels of correlates of conscious awareness, i.e., at the cellular level in the megahertz range and at the multicellular organization level with the synchronized and modulated firing (beat frequency) rate [1$100 \mathrm{~Hz}]$ of ensembles of neurons (they are called conscious moments in the Orch OR theory). The latter correlate of conscious awareness realizes the unity and binding of conscious content in the beat frequency rate, which is recorded in the EEG, fMRI and MEG signals [5], see Figure 2.

Such an approach is able to explain some phenomena, i.e., high activation in the $\beta$ and $\gamma$ spectral ranges during active thinking, focus, alertness and complex cognitive functions. In these mental states, the mind-field has to focus and interconnect multiple complex external mental objects into a meaningful and comprehensive system. That is, during this process, a self-centered mind-field, represented by the primary quantum state $\Psi_{p}$, expands to the external layers of the environment and is reflected back in the form of the quantum state $\Psi_{r}\left(\Psi_{r}^{m}, \Psi_{r}^{b}, \Psi_{r}^{s}\right)$. The reflected state $\Psi_{r}$ undergoes comparatively larger changes, because it reaches the outer external layers, which can be considerably and continuously affected by the volatile external environment.

Considering the above-mentioned conceptual ideas, by and large, the dynamics of the mind-field states can be described using an iterative mental cycle:

1. For a chosen time moment $t$, the mind-field initiates its primary (referential) and stationary quantum state $\Psi_{p}(t)$.

2. Part of $\Psi_{p}(t)$ interacts with the heterogeneous environment $\varepsilon$ (it includes external stimuli, the inner senses of the body and even the mind state as the deepest internal environment), which produces reflected nonstationary quantum state $\Psi_{r}(t)$.

3. The superposition of the primary $\Psi_{p}$ and reflected $\Psi_{r}(t)$ quantum states produces superposed quantum state $\Psi(t)=\Psi_{p}(t)$ $+\Psi_{r}(t)$; it forms a 3D spatial hologram within the mind-field.

4. The 3D spatial hologram of the mind-field affects the brain's complex neural network, leaving an energy imprint.

5. The brain-body consciously or unconsciously reacts to the induced energy imprint, which can involve the activation of the body through the peripheral nervous system using genetically inherited and learned psychosomatic connections.

6. After the procession of the above described chain of events (primal quantum state $\rightarrow$ reflected quantum state $\rightarrow$ superposed quantum state $\rightarrow$ spatial $3 \mathrm{D}$ hologram in the 
mind-field $\rightarrow$ energy imprint on the brain's neural networks $\rightarrow$ bodily response), one or another response from the environment follows.

7. The mental cycle repeats for the next time moment $t+1$.

\section{CONCLUSION}

This paper provides a putative link between the Orch OR theory and OAM based on the OSIMAS framework, and the empirically observed coherent bioelectromagnetic oscillations of ensembles of neurons in the brain (the EEG spectra). It also sheds new light on the novel interpretation of the Orch OR theory of quantum beats. In this short work-in-progress report, the proposed multidisciplinary approach provides conceptual tools for the modeling of the new type of agency.

Let us see some resulting examples. The superposition of differences between primary quantum state $\Psi_{p}$ and its reflections from the heterogeneous environment $\left(\Psi_{r}^{p}, \Psi_{r}^{b}, \Psi_{r}^{s}\right)$ generates beat frequencies $\omega^{m}, \omega^{b}$ and $\omega^{s}$ respectively. According to the Orch OR, they denote a decoherence rate, which naturally tends to be faster in the outer layers of the mind-field (see Figure 2), where environmental changes take place. Meanwhile, in the more centered layers of the mind-field, the need for a tuning process diminishes, e.g., in a state of the deep sleep, the mind-field is totally withdrawn from the senses (i.e., from the external environment) and is self-centered so that the primary quantum state $\Psi_{p}$ is not affected. In the case of deep sleep, the superposition of $\Psi_{p}$ with its very close reflection $\Psi_{r}$ produces a very low rate of beat frequencies. Therefore, socalled delta brainwaves $(0-4 \mathrm{~Hz})$ are observed during deep sleep.

High activation in the $\beta$ and $\gamma$ spectral ranges is correlated with active thinking, focus, alertness and complex cognitive functions. In these mental states, the mind-field has to focus and interconnect multiple complex external mental objects into a meaningful and comprehensive system. That is, during this process, the mind-field, represented by the primary quantum state $\Psi_{p}$, expands to the external layers of the environment and is reflected back in the form of the quantum state $\Psi_{r}\left(\Psi_{r}^{m}, \Psi_{r}^{b}\right.$, $\left.\Psi_{r}^{s}\right)$. The reflected state $\Psi_{r}$ undergoes comparatively larger changes, because it reaches the outer external layers, which can be considerably and continuously affected by the volatile external environment.

\section{ACKNOWLEDGMENT}

This research project is funded by the European Social Fund under the Global Grant measure; project No.VP1-3.1SMM-07-K-01-137.

\section{REFERENCES}

[1] D. Plikynas, A. Raudys, and S. Raudys, "Agent-based modelling of excitation propagation in social media groups," J. Exp. Theor. Artif. Intell., vol. 0, no. 0, pp. 1-16, 2014.

[2] D. Kezys and D. Plikynas, "Prognostication of Human Brain EEG Signal Dynamics Using a Refined Coupled Oscillator Energy Exchange Model," Neuroquantology, vol. 12, no. 4, pp. 337-349, 2014.

[3] D. Plikynas, "A virtual field-based conceptual framework for the simulation of complex social systems," J. Syst. Sci. Complex., vol. 23, no. 2, pp. 232-248, Apr. 2010.

[4] D. Plikynas, "Oscillating agent model: quantum approach,"
NeuroQuantology Press, 2015.

[5] D. Plikynas, G. Basinskas, P. Kumar, S. Masteika, D. Kezys, and A. Laukaitis, "Social systems in terms of coherent individual neurodynamics: conceptual premises, experimental and simulation scope,” Int. J. Gen. Syst., vol. 43, no. 5, pp. 434-469, Feb. 2014.

[6] D. Plikynas, G. Basinskas, and A. Laukaitis, "Towards oscillations-based simulation of social systems: a neurodynamic approach," Connect. Sci., vol. 0, no. 0, pp. 1-24, 2014.

[7] H. Atmanspacher, "Quantum Approaches to Consciousness," in The Stanford Encyclopedia of Philosophy, Summer 2011., E. N. Zalta, Ed. 2011.

[8] D. K. F. Meijer and K. Jakob, "Quantum modeling of the mental state: the concept of a cyclic mental workspace," Syntropy, vol. 1, pp. 1-41, 2013.

[9] H. $\mathrm{Hu}$ and $\mathrm{M}$. Wu, "Current Landscape and Future Direction of Theoretical \& Experimental Quantum Brain/Mind/Consciousness Research,” J. Conscious. Explor. Res., vol. 1, no. 8, Nov. 2010.

[10] S. Tarlac1, "A Historical View of the Relation Between Quantum Mechanics and the Brain: A NeuroQuantologic Perspective," NeuroQuantology, vol. 8, no. 2, Mar. 2010.

[11] A. Vannini and U. Di Corpo, "Quantum models of consciousness," Quantum Biosyst., vol. 2, pp. 165-184, 2008.

[12] D. Plikynas, G. Basinskas, and A. Laukaitis, "Towards oscillations-based simulation of social systems: a neurodynamic approach," Connect. Sci.

[13] S. Hameroff and R. Penrose, "Consciousness in the universe: A review of the 'Orch OR' theory," Phys. Life Rev., vol. 11, no. 1, pp. 39-78, Mar. 2014. 